\title{
Introduzione. Gli usi sociali del tempo libero: economie della condivisione nella crisi
}

Valentina Lusini, Pietro Meloni e Francesco Zanotelli

\section{OpenEdition}

1 Journals

Edizione digitale

URL: http://journals.openedition.org/aam/1352

DOI: $10.4000 /$ aam.1352

ISSN: 2038-3215

Editore

Dipartimento Culture e Società - Università di Palermo

Notizia bibliografica digitale

Valentina Lusini, Pietro Meloni e Francesco Zanotelli, «Introduzione. Gli usi sociali del tempo libero: economie della condivisione nella crisi », Archivio antropologico mediterraneo [Online], Anno XXII, n. 21

(1) | 2019, online dal 30 juin 2019, consultato il 23 septembre 2019. URL : http:// journals.openedition.org/aam/1352; DOI : 10.4000/aam.1352

Questo documento è stato generato automaticamente il 23 septembre 2019.

Archivio antropologico mediterraneo 


\title{
Introduzione. Gli usi sociali del tempo libero: economie della condivisione nella crisi ${ }^{1}$
}

\author{
Valentina Lusini, Pietro Meloni e Francesco Zanotelli
}

\section{Tempo libero impegnato}

1 Questa raccolta riunisce alcuni degli interventi presentati nel dicembre 2017 in occasione del V Convegno Nazionale della Società Italiana di Antropologia Applicata sul tema Collaborazione e mutualismo. Pratiche trasformative in tempo di crisi nell'ambito del quale abbiamo coordinato una sessione dal titolo "Gli usi sociali del tempo libero: pratiche di consumo di fronte alla crisi". La sessione aveva un carattere interdisciplinare, riunendo contributi di ricercatori antropologi, sociologi ed esperti di psicologia e del servizio sociale, con una particolare attenzione alla sfera applicativa. Il confronto in sede di discussione dei diversi interventi ha rappresentato un momento importante di condivisione di saperi, metodi e linguaggi.

2 La disponibilità di Archivio Antropologico Mediterraneo permette di ripresentare qui alcuni dei risultati di quella riflessione, con i contributi più significativi che sono stati sottoposti a un processo di selezione rigoroso, capace di soddisfare i requisiti di una rivista di settore che ha come principale obiettivo quello di far circolare tra gli specialisti, perlopiù accademici, i risultati di ricerche con un solido impianto teorico e un'impostazione apertamente etnografica. Questo processo, se ha avuto il pregio di garantire la qualità scientifica dei saggi accettati e pubblicati, ha tuttavia escluso i lavori ancora in corso di perfezionamento e quelli che, per il diverso approccio epistemologico e metodologico, si collocavano in territori disciplinari discontinui.

3 Le nozioni chiave del convegno, 'crisi' e 'mutualismo', sono argomenti essenziali della contemporaneità. La crisi, innanzitutto: economica, certo, ma anche migratoria, umanitaria, istituzionale, ambientale e, ancora, crisi di valori, crisi di identità, crisi dei confini disciplinari. La nozione di crisi sembra essere pervasiva in molti campi, da 
quello finanziario a quello politico, da quello cognitivo a quello culturale. L'uso che facciamo di questo termine può dirci molto sul nostro immaginario esistenziale, sul modo in cui interpretiamo le contraddizioni del nostro tempo. Nell'ambito delle riflessioni sviluppate durate la sessione, la nozione di crisi è valsa a interrogare il contenuto e la funzione del secondo argomento, il mutualismo, che abbiamo scelto di declinare come sociabilità, ovvero come particolare sensibilità verso l'altro che si configura come disposizione etica, come pratica e competenza sociale nel creare legami. Un concetto che, in questa forma, è stato tematizzato dal sociologo tedesco Georg Simmel (1917, tr. it. 2012²) e recentemente rielaborato da Richard Sennett in un denso libro sulle forme, i rituali, i piaceri e le pratiche della collaborazione (Sennett 2012).

4 Il tema attorno al quale abbiamo scelto di inquadrare le nozioni di crisi e sociabilità riguarda il tempo libero, inteso come cornice di attivazione di nuove forme di produzione, scambio e consumo orientate alla sperimentazione di reti di condivisione in contesti caratterizzati dal mutamento, dall'incertezza e dal misconoscimento sociale. Edgar Morin (2005) ebbe a scrivere che il tempo libero è un tempo guadagnato sul lavoro e orientato verso il loisir, lo spazio di evasione e intrattenimento che l'uomo medio pratica collocando se stesso nella cultura di massa. Si tratta, a ben vedere, di una concezione storicamente e culturalmente situata, che non corrisponde al modo in cui molte società concepiscono e vivono il tempo (Tentori 1999: 70). Quello che si osserva oggi, in particolare, è l'emergere di una nuova concezione di "tempo libero impegnato", un contesto di pratiche di sociazione (Simmel 2012) che associano ai principi dello svago e dell'autorealizzazione i valori della vita collettiva sublimata nella solidarietà sociale. La trasformazione del valore e dell'esperienza del tempo libero si può interpretare certo come conseguenza della condizione di deprivazione materiale che produce isolamento e disuguaglianza sociale, ma anche, seguendo Sennett, come risultato delle modificazioni del lavoro, sempre più flessibile, precario o part-time $\mathrm{e}$ sempre più caratterizzato da relazioni sociali a breve termine e raramente orientate alla costruzione di legami stabili. In un contesto di questo tipo, in cui la collaborazione è dequalificata sia sul piano delle attività produttive sia su quello delle reti sociali, l'esigenza di collaborare si fa più necessaria (Sennett 2012).

5 Come afferma Arjun Appadurai (2013), abbiamo oggi la necessità di pensare al futuro come a un orizzonte culturale alimentato dalle aspirazioni, dalle proiezioni e dall'immaginazione degli attori sociali, tutti aspetti che assumono grande importanza nell'ottica delle pratiche dell'economia circolare. Si pensi ad esperienze come quella di Solidarity for All, che in Grecia propone un modello di mutualismo che favorisce lo sviluppo della cultura dell'auto-organizzazione e della solidarietà, creando strumenti e spazi comuni di economie alternative. Si pensi anche alle molte esperienze di agricoltura sociale e gestione condivisa di aree rurali abbandonate e residuali, che rispondono alle condizioni di vulnerabilità e incertezza occupazionale promuovendo forme di produzione e consumo alimentare improntate all'utilità sociale e al rilancio economico delle produzioni tipiche. Si pensi alle svariate forme dell'economia senza intermediari, alle offerte del turismo e della mobilità organizzate sull'home-sharing, sul car-pooling o sul social-eating. E, ancora, si pensi alle numerose esperienze di commercio senza uso di denaro o alle piattaforme online che utilizzano le potenzialità della rete per mettere in contatto persone che si scambiano competenze, servizi, tempo, disponibilità e beni. Si tratta di contesti dove funziona la collaborazione dialogica 
(Sennett 2012: 144), che si avvale dell'empatia finalizzata al vantaggio reciproco, all'essere insieme nella complessità e nella differenza.

6 In tempi di incertezza economica e sociale, un dono moderno tra estranei (Godbout 1993) può esprimersi in competenze relazionali che spesso prendono vita dal basso. $\mathrm{Si}$ prenda il caso del dono del proprio tempo (Derrida 1996), concepito come eccedenza che può attivare un circolo di reciprocità in senso maussiano. Da questo punto di vista le iniziative mutualistiche delle cooperative di consumo, le associazioni e i centri culturali solidali, i progetti di solidarietà alimentare e cittadinanza attiva, le azioni partecipative di qualificazione della produzione in una direzione di sostenibilità, sono esempi di situazioni in cui la condivisione di progetti, oggetti, spazi e tempi di socialità non è solo principio etico alternativo di consumo (Bauman 2010), ma strategia di resistenza e sopravvivenza, risposta ai bisogni legati alla necessità di ripensare in modo consapevole e critico l'uso delle risorse, non solo materiali, gli stili di vita e le scelte del vivere quotidiano.

\section{Serio ludere}

7 Se l'idea di tempo libero sta mutando, le direzioni intraprese da diversi attori sociali riguardano soprattutto gli aspetti di scambio e di condivisione. Come spendere il tempo che abbiamo a disposizione al di fuori del lavoro in modo che possa essere ritenuto eticamente e socialmente accettabile? Se il tempo libero della cultura di massa, in quanto risultato del lavoro salariato e dell'affermazione del ceto impiegatizio, è alimentato dall'esibizione di attività evasive e ludiche (vacanze moderne, week-end, cene del fine settimana, sport di spiaggia ecc.) che sottolineano la discontinuità tra lavoro e vita quotidiana (Morin 2005: 75), il tempo libero contemporaneo, per quanto non perda queste caratteristiche, si arricchisce della necessità di costruire reti sociali, di sviluppare coscienze ecologiste, di assumere una postura riflessiva nei confronti del mondo quotidiano. Gli articoli proposti, in modi tra loro assai differenti, rispondono a questa mutata sensibilità da parte degli attori sociali nei confronti dell'uso del tempo.

8 Il testo di Viola Lucrezia Giuliani, Una seconda opportunità per le cose. Circuito del dono e processi di mercificazione in un emporio dello scambio e del baratto, affronta un tema di grande rilievo che incrocia le pratiche di consumo, alcuni principi dell'economia circolare e il ruolo degli oggetti nella società di oggi. Partendo dall'analisi etnografica di un emporio dell'usato a Orvieto, il "Senza Moneta", l'autrice discute le motivazioni etiche che hanno spinto all'apertura e alla gestione di questa attività, le pratiche dei donatori e dei consumatori e il funzionamento di un luogo dove si è deciso di bandire l'uso del denaro in favore di un baratto mediato da monete simboliche - acquistabili esclusivamente mediante donazione di cose. Questo meccanismo rende il "Senza Monete" un luogo dove il baratto è fortemente influenzato dalle logiche del dono e dove il tempo libero viene riconfigurato a partire dall'idea di scambiare cose con degli sconosciuti non solo per il piacere di farlo, ma nella convinzione che la donazione e lo scambio consentano di ridurre la disparità sociale ed economica tra persone.

9 L'articolo di Giorgio Cassone, Lavoro, consumo e tempo libero nelle pratiche informali di riciclo alimentare. Il reciclaje nella città di Granada (Spagna), è incentrato sulle pratiche di riciclo alimentare a Granada. Il reciclaje è l'attività di recupero di scarti alimentari dai cassonetti presso i mercati cittadini, le panetterie, i grandi supermercati. L'autore definisce questa attività come una forma di lavoro che si attua nel tempo libero e che 
riguarda diverse categorie di persone: poveri, anziani, gitani, studenti. Dall'indagine etnografica emerge come questa attività non sia legata soltanto a necessità economiche, contingenti o strutturali, ma implichi anche un'attenzione verso le forme del riciclo, del non spreco, della decrescita (Latouche 2008). Il reciclaje si configura, in questa prospettiva, come una forma di condivisione di alimenti destinati allo smaltimento (chi ricicla raramente consuma da solo) che ridefiniscono gli spazi di vita quotidiana e l'uso del proprio tempo libero, ricalibrato sugli orari di chiusura dei negozi e dei mercati e sui luoghi dove si può praticare in modo fruttuoso il riciclo alimentare.

10 I temi degli altri due articoli spostano la questione del tempo libero, del dono e delle forme di condivisione su terreni diversi: quelli del gioco e dei social network. L'articolo di Federica Scrimieri, Giveaway. Per un'etnografia del dono nelle communities on line, presenta i risultati di una densa indagine etnografica sul mondo digitale, con un focus sul dono presso le comunità online presenti su YouTube e Facebook. Campo di indagine recente e in crescente aumento, l'antropologia digitale (Miller, Horst 2013; Hine 2015) e dei social media (Miller 2016; Miller et al. 2018) ci aiuta a comprendere nuove forme di socializzazione e, nello specifico del contributo di Scrimieri, quali forme assume un tema classico negli studi antropologici come quello del dono. L'autrice si interroga infatti sull'emergere di pratiche che sembrano non rispondere all'immagine individualistica ed edonistica che spesso ci viene restituita dei social media e del consumo (Lipovetsky 2007). Il dono fra sconosciuti senza un reale interesse - salvo forse il disfarsi di oggetti di cui non si ha più bisogno - è una pratica che ha trovato una ridefinizione attraverso il mondo digitale. Il dono finalizzato alla costruzione di una rete di followers permette agli influencers di ampliare il proprio capitale sociale (Bourdieu 2011) in modo molto più veloce rispetto al passato. Il tempo libero impiegato online sembra avere lo scopo preciso di cambiare qualcosa nella vita quotidiana degli attori sociali mediali.

11 L'ultimo articolo è a firma di Aurelio Castro, che in Playing is sharing: la condivisione e il tempo libero nelle reti sociali di gioco esamina il tempo libero come un fenomeno non contrapposto al tempo del lavoro, ma come uno spazio autonomo da analizzare con categorie interpretative diverse e specifiche. La riflessione dell'autore parte da un'etnografia che incrocia saperi antropologici e psicologici, facendo largo uso dell'osservazione partecipante come metodologia capace di rendere conto della profondità dei giochi e della serietà con la quale le comunità di gioco pensano se stesse. La ricerca restituisce un contesto in cui la condivisione di spazi di gioco trasforma il tempo libero in tempo fortemente qualitativo, capace di produrre una coesione informale che ridefinisce relazioni sociali e coordinate della comunicazione con ricadute sull'equilibrio emotivo e sulle dimensioni del sentimento e del desiderio.

\section{Sperimentare la condivisione}

12 I quattro contributi di ricerca offerti ci restituiscono, nella diversità degli approcci metodologici e dei casi di studio, un panorama contemporaneo nel quale le persone, accomunate dall'incertezza economica ed esistenziale dei tempi che viviamo, creano spazi di socialità, inventano forme di condivisione, praticano in modo originale il consumo, a volte con intenzioni trasformative e di contestazione dell'esistente. Inoltre, la sensazione che traspare dai testi che presentiamo è che tale creatività, sebbene non 
sia sempre accompagnata dalla convivialità (Scrimieri) auspicata da Latouche (2008), sia comunque fonte di piacere, fino a sfociare apertamente nel gioco (Castro).

13 Bisognerebbe indagare maggiormente il nesso tra piacere ed economia dello scambio e, piuttosto che riferirsi alla «passione calma del far danaro» che Hirschmann (2011) associa alle origini del capitalismo, nell'attuale epoca di crisi del turbo o finanzcapitalismo (Gallino 2013), e anche orientare la nostra attenzione verso le passioni che spingono le persone a rimettere in circolo (Giuliani), a recuperare e trasformare (Cassone) beni che, dopo essere stati originati in qualità di merci, lo stesso circuito dell'economia mercantile blocca o espelle. Come insegna Kopytoff (1986), la possibilità che tali cose assumano nuova vita passa per il loro trasferimento, al contempo simbolico e materiale, in regimi economici diversi che qui vengono individuati nel dono, nel baratto, nella condivisione.

14 Emerge, poi, un ulteriore elemento di notevole interesse teorico che potremmo sintetizzare nell'idea che esiste una connessione tra i processi di shifting context appena citati e le potenzialità trasformative delle tattiche (de Certeau 2001) che le persone mettono in campo nel praticare comportamenti paralleli o addirittura apertamente oppositivi a quelli dell'acquisto mercantile e del lavoro salariato. Tutti i saggi mostrano infatti che le pratiche analizzate, siano esse il giocare in gruppi organizzati, il riciclare cibo-spazzatura, il barattare e donare vestiti e oggetti in spazi fisici preposti oppure online, sono accomunate dal fatto che esse hanno luogo (e tempo) in una condizione liminale, caratterizzata dall'ambigua compresenza o, potremmo dire, dalla condizione in bilico e di continuo passaggio dall'interno del sistema di produzione e scambio capitalistico verso il suo esterno.

15 Questa condizione è particolarmente evidente a partire dalle modalità con le quali i protagonisti delle ricerche qui presentate concepiscono il tempo - sottratto, più che liberato - alle usuali relazioni di lavoro e dedicato alle azioni documentate. Come abbiamo già ricordato sopra, esso non viene infatti ascritto al tempo del loisir; al contrario, si tratta di un tempo considerato estremamente produttivo, ma nell'ambito delle relazioni sociali. L'ambiguità, o meglio l'oscillazione, risulta dal fatto che il tempo può venir finalizzato in alcuni casi all'acquisizione di maggiori competenze da "giocarsi" nuovamente nella sfera lavorativa (Castro; Scrimieri); in altri casi è invece più nettamente orientato a costruire alternative esistenziali, allontanandosi, seppur parzialmente, dalla sfera di scambio e dalla produzione incentrate prevalentemente sulle relazioni sociali di tipo capitalistico (Cassone; Giuliani).

16 Una questione che le quattro ricerche aprono, e che per forza di cose non risolvono, riguarda la potenzialità trasformativa in senso strutturale di tali esperienze. Esse ci spingono, indirettamente, a riesumare il concetto polanyiano di "processo di istituzionalizzazione dell'economia", che si basa sostanzialmente sui processi storici di integrazione tra specifiche forme dello scambio, strutture ideo-logiche e organizzazioni sociopolitiche. La lezione di Polanyi (1980) ci dovrebbe pertanto indurre a osservare i comportamenti economici in fieri, oltre che quelli embedded; quelli marginali, ma potenziali, oltre che quelli già strutturati e istituzionalizzati (Graeber 2012). Una lezione che già Alfredo Salsano (2008) aveva declinato al presente, introducendo l'idea che le forme dello scambio economico, piuttosto che alternarsi in fasi storiche separate, convivessero in una sorta di "poligamia" gerarchicamente organizzata, ma potenzialmente riformulabile. Recentemente, Peebles (2015: 480) ha sottolineato quanto, nel dibattito sullo scambio in antropologia economica, si sia andata 
consolidando l'idea che la contrapposizione binaria tra categorie (merci/doni; credito/ debito; stato/mercato, ecc.) debba piuttosto essere sostituita da un'indagine sulla costruzione storico-ideologica di tali opposizioni.

17 Tra le varie modalità di economia sociale, quella che sta emergendo maggiormente all'attenzione recente degli economisti, dopo esser stata introdotta (e poi trascurata) dagli antropologi specializzati nelle società di caccia e raccolta, è la condivisione (Aria 2016: 145 e passim). Ma se alla contemporanea sharing economy associamo piattaforme on-line, pratiche collaborative, relazioni orizzontali e reputazione digitale, una riflessione più approfondita, che parta dal significato etimologico del termine, ci permetterà di concludere questa presentazione dei contributi di ricerca evidenziando un ulteriore comune tratto che li caratterizza. To share assume in inglese il doppio significato sia di "spartizione" (dividere, ridurre in parti) che di "condivisione" (prendere parte, partecipare). Tale unità lessicale dà l'idea che il momento della spartizione e quello della condivisione siano due movimenti dello stesso processo. Per spartire è necessario condividere, così come senza un reciproco e comune soddisfacimento non c'è condivisione. Nella raccolta di casi etnografici che proponiamo, la condivisione emerge non in qualità di categoria economica definita, piuttosto assume i tratti di un movimento sfumato in grado però di raccogliere un insieme di pratiche diverse (dono anonimo, baratto, riciclo, riuso, gioco in comune, addirittura alcune espressioni del mercato) sotto una medesima tensione: di fronte a un sistema capitalistico de-socializzante, acuito dalla crisi, una possibilità sta nella messa in comune di tempo, risorse e creatività. Similmente a quanto succede per le società acquisitive, che nei momenti di eccesso di risorse maggiormente esprimono la propensione (e l'obbligo) alla spartizione (Solinas 1992: 90), così nella nostra società estremamente stratificata, l'opulenza e l'eccesso di risorse circolanti nel mercato producono in alcuni la necessità di inventare pratiche di spartizione e di condivisione.

\section{BIBLIOGRAFIA}

Appadurai A., 2013 Il futuro come fatto culturale. Saggi sulla condizione globale, Raffaello Cortina, Milano.

Aria M., 2016 I doni di Mauss. Percorsi di antropologia economica, Cisu, Roma.

Bauman Z., 2010 L'etica in un mondo di consumatori, Laterza, Roma-Bari.

Bourdieu P., 2011, La distinzione. Critica sociale del gusto, il Mulino, Bologna.

de Certeau M., 2001 L'invenzione del quotidiano, Edizioni Lavoro, Roma.

Derrida J., 1996 Donare il tempo. La moneta falsa, Cortina, Milano.

Gallino L., 2013 Finanzcapitalismo. La civiltà del denaro in crisi, Einaudi, Torino.

Godbout J. T., 1993 Lo spirito del dono, Bollati Boringhieri, Torino.

Graeber D., 2012 La rivoluzione che viene. Come ripartire dopo il capitalismo, Manni, Lecce. 
Hine Ch., 2015 Ethnography for the Internet, Bloomsbury, London.

Hirschman A.O., 2011 Le passioni e gli interessi. Argomenti politici in favore del capitalismo prima del suo trionfo, Feltrinelli, Milano.

Kopytoff I., 1986 «The cultural biography of things: commoditization as process», in Appadurai A. (ed.), The Social Life of Things: Commodities in Cultural Perspective, Cambridge University Press, Cambridge: 64-92.

Latouche S., 2008 Breve trattato della decrescita serena, Bollati Boringhieri, Torino.

Lipovetsky G., 2007 Una felicità paradossale. Sulla società dell'iperconsumo, Raffaello Cortina, Milano.

Mauss M., 2000 «Saggio sul dono. Forma e motivo dello scambio nelle società arcaiche», in Teoria generale della magia e altri saggi, Einaudi, Torino: 155-269.

Miller D., Horst H. (eds), 2013 Digital Anthropology, Bloomsbury, London.

Miller D., 2016 Social Media in an English Village, UCL, London.

Miller D. et al., 2018 Come il mondo ha cambiato i social media, Ledizioni, Milano.

Morin E., 2005 Lo spirito del tempo, Meltemi, Roma.

Polanyi K., 1980 Economie primitive, arcaiche e moderne, Einaudi, Torino.

Salsano A., 2008 Il dono nel mondo dell'utile, Bollati Boringhieri, Torino.

Sennett R., 2012 Insieme. Rituali, piaceri, politiche della collaborazione, Feltrinelli, Milano.

Simmel G., 2012 La socievolezza, Armando Editore, Roma.

Solinas P.G., 1992 Economie minori. Saggi di antropologia, Edes, Sassari.

Tentori T., 1999 Antropologia delle società complesse, Armando, Roma.

\section{NOTE}

1. La presente introduzione così come l'ideazione e la cura della sezione speciale sono il frutto della condivisione e del lavoro congiunto dei tre autori. La stesura materiale dei paragrafi è da attribuire a Lusini (\$1), Meloni (\$2) e Zanotelli (\$3).

\section{AUTORI}

\section{VALENTINA LUSINI}

Università degli Studi di Siena lusiniva@unisi.it

\section{PIETRO MELONI}

Università degli Studi di Siena pietro.meloni@unisi.it 


\section{FRANCESCO ZANOTELLI}

Università degli Studi di Messina francesco.zanotelli@unime.it 